\title{
Design of an Antenna Array for GNSS/GPS Network
}

\author{
Hocine Hamoudi ${ }^{1,2}$, Boualem Haddad ${ }^{2}$, and Philippe Lognonné ${ }^{3}$ \\ ${ }^{1}$ National Institute of the Post and I.C.T, Box 156, 16220 Eucalyptus, Algiers, Algeria \\ h_hamoudi@inptic.edu.dz \\ ${ }^{2}$ University of Sciences and Technology Houari Boumedien, Laboratory of Image Processing \\ and Radiation, Box 32, 16111 El Alia, Bab Ezzouar, Algeria \\ bhaddad_57@yahoo.fr \\ ${ }^{3}$ Institut de Physique du Globe de Paris (IPGP), 94107 Saint Maure, France \\ lognonne@ipgp.fr
}

\begin{abstract}
This work focuses precisely on the design of a smart antenna printed on dielectric substrate operating at frequency $\mathrm{L} 1=1575.42 \mathrm{MHz}$ This device consists of an antenna array to be integrated, in a GNSS/GPS network, with the aim of detecting ionosphere disturbances associated with land-based. To address such concerns, we studied an antenna array, consisting of four square elements, patch type, operating at the L1 frequency. As a first step, a simple square printed radiating structure was designed to test adaptation and radiation characteristics. In a second step, a square shape $(2 * 2)$ antenna array has been studied. This type of sensor (networks) should respond no later than fifteen minutes after the main shock of an earthquake.
\end{abstract}

Keywords: Antenna array, GNSS antenna, ionosphere perturbations.

\section{Introduction}

At the end of the last decades, significant progress in detecting and modeling the ionosphere disturbances induced by seismic waves, were performed. This research is now an important part of the assignment and monitoring projects in the upper atmosphere [1] [2]. We are particularly interested in the behavior of the ionosphere, which is considered the seat of physical phenomena such as refraction and reflection of electromagnetic waves.

The extremely low power level delivered at the satellite makes the GPS prone to jamming and interference disturbances. Interference in question may be unintended equipment produced by other radio or from hostile interference. Studies by [3] and [4] show that an antenna array and adaptive directional beams are a promising method to overcome this problem. The use of high frequencies and microwave systems for microstrip structure has been responsible for the development of printed antennas. They are most often used in networks to improve performance and to enable the achievement of very specific functions, such as pointing and scanning electronic jammers rejection, and adaptive detection. There are two possible methods to dynamically change the radiation pattern and mitigate the effects of interference and multipath while increasing the coverage and distance [3]: (1) The switched beam and 
(2) the adaptive beamforming. However, the first system provides limited performance compared to the second one. Therefore, we will focus our work on adaptive beam antennas which can react dynamically to changing RF environment. The unwanted signals (interferences) are deleted, while the main beam is directed to the signal. It is precisely to meet these requirements we are considering to use the antenna arrays controlled by servo. The servo leads the radiation beam to point to a desired mobile user and tracks the moving user, while minimizing interference from other users. Precisely, a part of this result we will try to achieve, in this study for a design in the industry, a network of smart antennas. The present work will be divided into two main parts. The first one will be devoted to the study of a single element (patch) square, while the second part, will addresses the computations and optimization of an antenna array.

\section{Simple Element Design}

The probably most two popular GNSS L1 antenna implementations are the patch and helix approaches. Nevertheless, others also exist [5]. This patch will be fed by a microstrip line with an impedance of $50 \Omega$. The study of such a unit cell allows us to observe their behavior and frequency radiation at the working frequency L1.

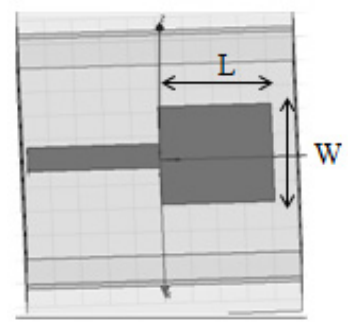

Fig. 1. Geometry of a single square patch

The described element will be design to operate at L1 frequency $1575.42 \mathrm{MHz}$. Our computations were based on transmission line theory. The width and length are given by the following equations [6]:

$$
\mathrm{W}=\frac{\mathrm{c}}{2 \mathrm{~L}_{1} \sqrt{\frac{\varepsilon_{\mathrm{r}}+1}{2}}}
$$

Where the operating frequency $\mathrm{L} 1=1575.42 \mathrm{MHz}, \mathrm{c}=310^{8} \mathrm{~m} / \mathrm{s}$ and $\mathrm{er}=4.32$. Substituting above values, the width of the patch (W) becomes $61.1 \mathrm{~mm}$. The effective length $\left(\mathrm{L}_{\text {eff }}\right)$ of the element can be calculated with equations (2) and (3).

$$
\mathrm{L}_{\mathrm{eff}}=\frac{\mathrm{c}}{2 \mathrm{~L}_{1} \sqrt{\varepsilon_{\mathrm{eff}}}}
$$


And

$$
\varepsilon_{\mathrm{eff}=} \frac{\varepsilon_{\mathrm{r}}+1}{2}+\frac{\varepsilon_{\mathrm{r}}-1}{2}\left(\frac{1}{\sqrt{1+12 \mathrm{~h} / \mathrm{W}}}\right)
$$

In this design, substrate has been used with parameters are: $\tan \delta=0.001$, thickness of substrate (h) equal to $1.59 \mathrm{~mm}$. Substituting $\mathrm{W}=61.1 \mathrm{~mm}, \varepsilon \mathrm{r}=4.32$ in equation (3), we get $\varepsilon_{\text {eff }}=4.10$. Hence $L_{\text {eff }}=49.9 \mathrm{~mm}$. Due to the board effect, we derive the length extension $(\Delta \mathrm{L})$, using the following equation :

$$
\Delta \mathrm{L}=0.412 \mathrm{~h} \frac{\left(\varepsilon_{\mathrm{eff}}+0.3\right)\left(\frac{\mathrm{W}}{\mathrm{h}}+0.264\right)}{\left(\varepsilon_{\mathrm{eff}}-0.258\right)\left(\frac{\mathrm{W}}{\mathrm{h}}+0.8\right)}
$$

Substituting $\varepsilon_{\text {eff }}=4.10$ and the values of $\mathrm{W}$ and $\mathrm{h}$, we get $\Delta \mathrm{L}=0.741 \mathrm{~mm}$. In final, we obtain, using this equation: $\mathrm{L}=\mathrm{L}_{\mathrm{eff}}-2 \Delta \mathrm{L}=49.9 \mathrm{~mm}-1.482 \mathrm{~mm}=48.41 \mathrm{~mm}$. However, some adjustments to the dimensions will be required in order to obtain a better adaptation and optimization of dimensions at the L1 frequency. The new dimensions of the patch, after optimization, are given in the following table.

Table 1. Dimensions of the patch

\begin{tabular}{ccc}
\hline Dimensions (mm) & Theoretical values & After optimization \\
\hline Length ( L) & $48.41 \mathrm{~mm}$ & $43 \mathrm{~mm}$ \\
\hline Width $(\mathrm{W})$ & $61.1 \mathrm{~mm}$ & $43 \mathrm{~mm}$ \\
\hline Thickness $(\mathrm{h})$ & $1.59 \mathrm{~mm}$ & $1.59 \mathrm{~mm}$ \\
\hline
\end{tabular}

The major characteristics, at the desired frequency of a single element, of adaptation and radiation are shown, respectively, in the Fig. 2, Fig.3, Fig.4 and Fig.5.

A good agreement is obtained at $1575.42 \mathrm{MHz}$ where the reflexion coefficient is about $-14 \mathrm{~dB}$. This value shows that the printed square patch antenna is better matched to its feeding strip line because $S_{11} \leq-10 \mathrm{~dB}$. In this investigation, VSWR is less than 2. Taking as a test transmission below $-10 \mathrm{~dB}$ to define the frequency at which the patch works (Fig. 2), there is then a cut ranging from $1.55 \mathrm{GHz}$ to 1.60 $\mathrm{GHz}$ - a bandwidth of about $4 \%$ compatible with the intended application. The antenna gain equal $4.91 \mathrm{~dB}$ in the direction of maximum radiation, that is perpendicular to the patch $0^{\circ}$. These results are satisfactory compared to the gain of patch antennas which seldom exceeds $6 \mathrm{~dB}$ [7]. 


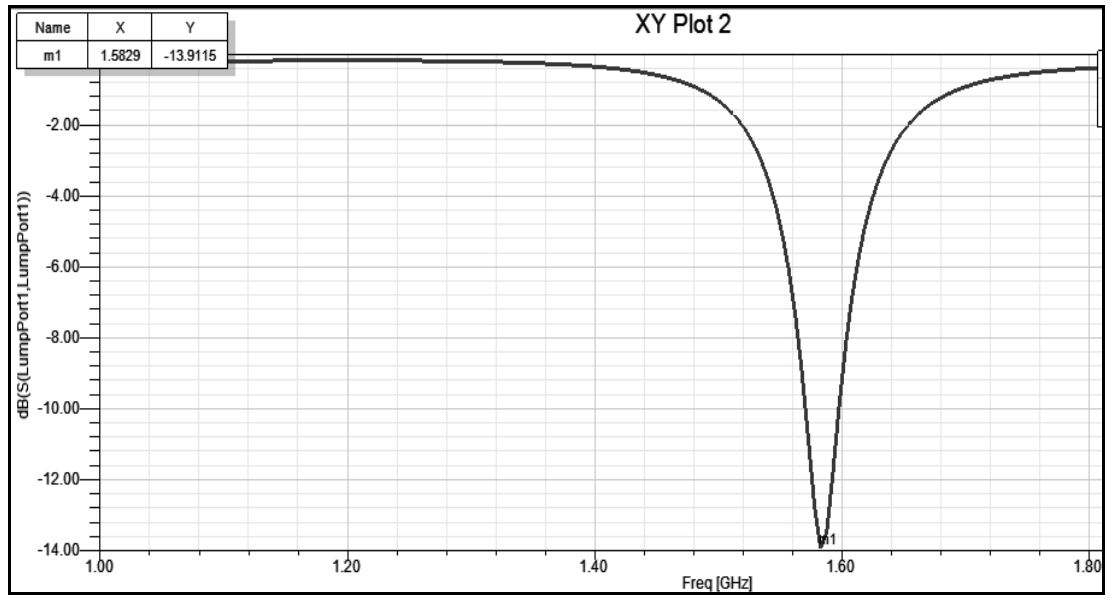

Fig. 2. Reflexion coefficient $\left(S_{11}\right)$ for single element

Taking as a test transmission below $-10 \mathrm{~dB}$ to define the frequency at which the patch works (Fig. 2), there is then a cut ranging from $1.55 \mathrm{GHz}$ to $1.60 \mathrm{GHz}$ a bandwidth of about $4 \%$ compatible with the intended application.

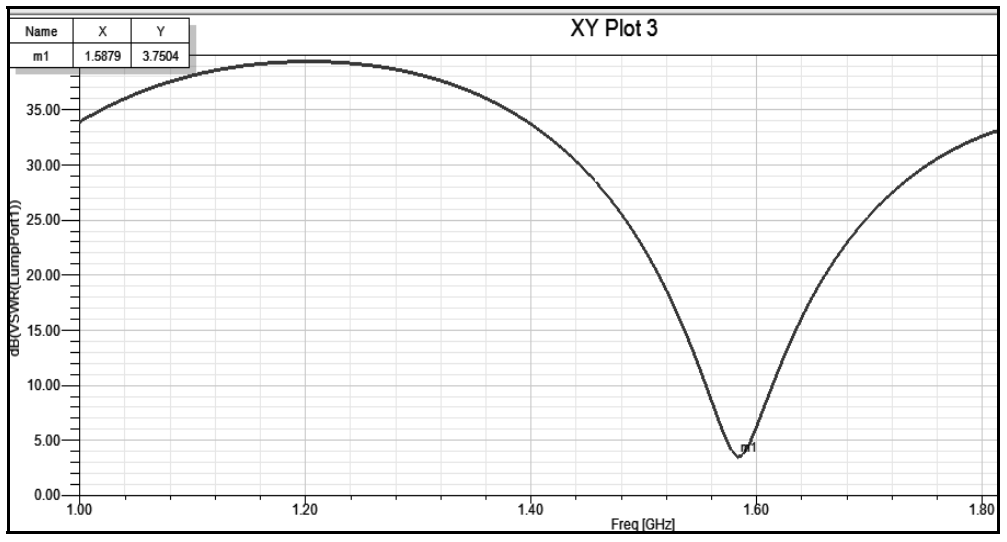

Fig. 3. VSWR for single element 


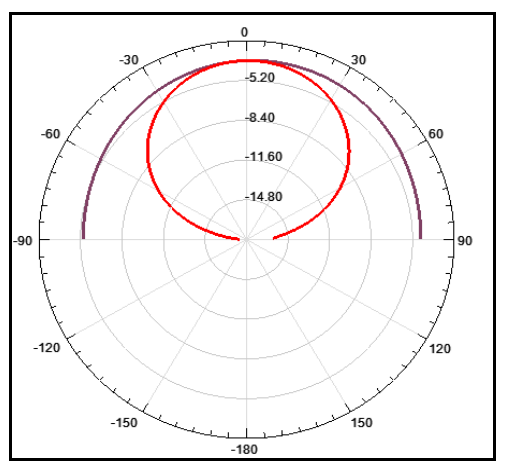

Fig. 4. Elevation pattern gain simulated for $\varphi=0^{\circ}$ (red) and $\varphi=90^{\circ}$ (purple)

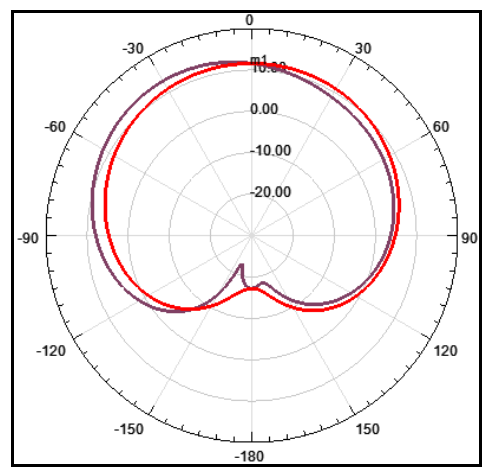

Fig. 5. Simulated radiation pattern at L1 frequency $\varphi=0^{\circ}$ (red) and $\varphi=90^{\circ}$ (purple)

\section{Antenna Array Design}

In the case of single square element, it has been observed that the antenna gain is quite low, not exceeding $6 \mathrm{~dB}$. In order to increase the gain and improve the radiation characteristics, we use a network of antennas instead of a single antenna element. The major advantage of this array is its electronic scanning capability. Moreover, the major lobe can be steered toward any direction by changing the phase of the excitation current at each array element.

The most important point in the design of an antenna array is the feed network. In our case, we opted for a parallel feed. The parallel feed, also called the corporate feed, where the patch elements are fed in parallel by the power division transmission lines. The transmission line divides into two branches and each branch divides again until it reaches the patch elements. This is constructed by first connecting two adjacent elements together with a transmission line and this can be calculated from (5) and (6). Now, two separate groups, each containing two elements, need to be connected together. This is done with a transmission line drawn between the centers of the 0.35 $\mathrm{mm}$ wide transmission line. Figure 6 show the geometry of the proposed array. 


$$
\mathrm{Z}_{0}=\frac{\eta_{0}}{\sqrt{\varepsilon_{\text {eff }}}}\left[\begin{array}{l}
\frac{\mathrm{W}_{\mathrm{e}}}{\mathrm{t}}+1.393+ \\
\left.0.667 \ln \left(\frac{\mathrm{W}_{\mathrm{e}}}{\mathrm{t}}+1.444\right)\right]
\end{array}\right]^{-1}
$$

Where

$$
\frac{\mathrm{W}_{\mathrm{e}}}{\mathrm{t}}=\frac{\mathrm{W}}{\mathrm{t}}+\frac{1.25}{\pi} \frac{\mathrm{h}}{\mathrm{t}}\left[1+\ln \left(\frac{4 \pi \mathrm{W}}{\mathrm{h}}\right)\right]
$$

Where $\mathrm{W}_{\mathrm{e}}$ is the effective width of the patch, $\mathrm{t}$ is the thickness of the dielectric substrate, $Z_{0}$ is the impedance of the transmission line and $\eta_{0}$ is the free space intrinsic impedance. The transmission line is split using $\mathrm{T}$-junction with equal power split. So both branches will receive input power, as is showing by the following equation:

$$
\mathrm{P}_{\text {in }}=\frac{1}{2} \frac{\mathrm{V}_{0}^{2}}{2 \mathrm{Z}_{0}}
$$

In that case where $\mathrm{P}_{\mathrm{a}}$ and $\mathrm{P}_{\mathrm{b}}$ be the output power, then

$$
\mathrm{P}_{\mathrm{a}}=\mathrm{P}_{\mathrm{b}}=\frac{1}{2} \mathrm{P}_{\text {in }}=\frac{1}{2} \frac{\mathrm{V}_{0}^{2}}{2 \mathrm{Z}_{\text {out }}}
$$

As equal power split is needed, the output impedance $\left(Z_{\text {out }}\right)$ of the transmission line using (7) and (8) is obtained as $Z_{\text {out }}=2 Z_{0}$. In general, the impedance of a patch is between 100 and $400 \Omega$ [8]. In our case, the transmission line is equal to $108 \Omega$ and knowing that the impedance $\mathrm{Z}_{\text {out }}=2 \mathrm{Z}_{0}$. Hence $\mathrm{Z}_{0}=54 \Omega$. We finally obtained a line width of $7 \mathrm{~mm}$. The array is fed by a probe of diameter $1.2 \mathrm{~mm}$ in the middle of the thicker transmission line by using SMA of impedance $50 \Omega$. From equation (8) we get that the probe ideally should have an impedance of $48 \Omega$.

In this method, the inner conductor of the coax is connected to the patch through the substrate while the outer conductor is attached to the ground plane.

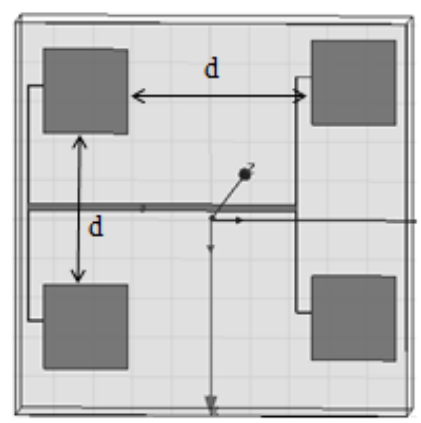

Fig. 6. Design of antenna array $(2 * 2)$ 
Particular interest should be worn to calculate the distance between element(s) in order to minimize the coupling between the elements. Several studies [8] and [9] showed that a distance of about $\lambda / 2$ reduces the effects of this phenomenon. Given that $\lambda$ is the wavelength in the dielectric, equal to $\lambda_{0} / \sqrt{ } \varepsilon_{\mathrm{eff}}$ with $\lambda_{0}$ the wavelength in vacuum and $\varepsilon_{\text {eff }}$ effective dielectric constant of the patch. In our case, the distance (d) between the elements of the antenna has been optimized and set at $8 \mathrm{~cm}$. This parameter has a significant impact not only on minimizing coupling phenomena but also the shape of the radiation pattern [4].

In future work, we will focus on the inter-element distance patches to study the influence of this parameter and optimize the performance of our network. The antenna is made of right hand circular polarization (RHCP) and it is on this basis that the antenna was carried out. The choice of this polarization is defined and not arbitrary [10].

The microstip antenna array radiates normal to its patch surface. The diagram consists essentially of a main lobe containing the maximum power, in the normal direction of the patch, suitable for our application. The simulated E-plane and H-plane pattern and the reflexion coefficient (S11) are illustrated in the figure 7 and 8 . The reflexion coefficient of the antenna array is $-14.82 \mathrm{~dB}$ at the $\mathrm{L} 1$ frequency for a peak gain at design frequency of $7 \mathrm{~dB}$.

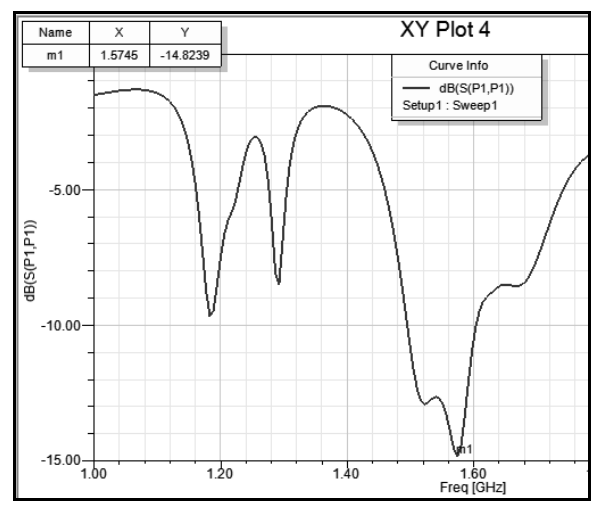

Fig. 7. Reflexion coefficient of the array

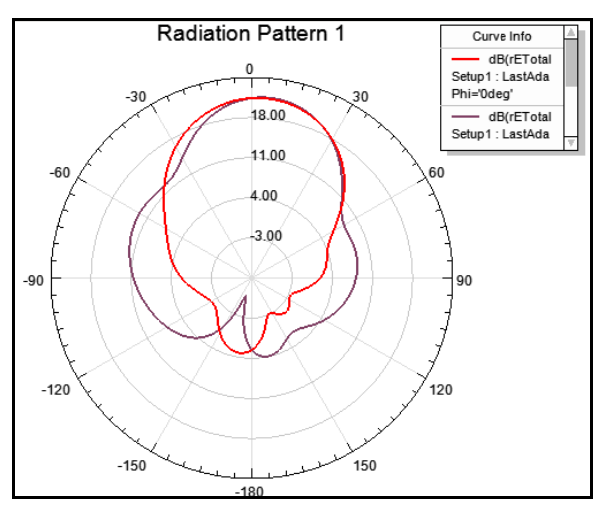

Fig. 8. Simulated E-plane and H-plane

\section{Conclusion}

The study of printed antennas shows that, we were able to design an antenna array consisting of four square elements, which will then be integrated into GPS/GNSS networks to monitor atmospheric phenomena. The results using simulation tools in the vicinity of $1547.42 \mathrm{MHz}$, showed satisfactory characteristics of adaptation and radiation. The appropriate approach to reach our goal is to study as a first step, and optimize the performance of a simple radiating component obtaining thus a reflexion coefficient close to $-15 \mathrm{~dB}$ with a gain of $5 \mathrm{~dB}$ and a suitable radiation pattern. Indeed, the maximum radiation is obtained in the normal direction of the patch. The results are fully consistent with the results in the literature. 
The second step consists in the optimization of antenna array by finding an optimal configuration of the network, addressing the key issue the distance between elements in order to obtain a small footprint while maintaining correct characteristics of adaptation and radiation. In this case, too, the radiation characteristics and adaptation remains satisfactory. We obtain a gain of about $7 \mathrm{~dB}$ and a radiation pattern consists mainly of a main lobe in the direction normal to the surface of the patch, suitable for our application. The inter-element distance was optimized and fixed to the half wavelength, about $8 \mathrm{~cm}$

In the case of single element it has been observed that the antenna gain is quite low. But, while employing the array, gain increases significantly. This is one of the most advantages of the array structure. The results obtained at the L1 frequency, make it possible to design a network of printed antennas that can be used further for the design of an adaptive antenna.

The network thus proposed with high gain, low cost and small footprint meets our goal. As a perspective to our work, we propose the use of this network for the design of an adaptive antenna capable to modify the antenna pattern in order to have the benefits of the signal environment. A beam adaptive antenna arrays using controlled by a well-defined process. This control directs the radiation beam to a desired mobile user and tracks the moving user, while minimizing interference from other users by introducing nulls in the direction of interference.

\section{References}

1. Lognonne, P., Artru, J., Garcia, R., Crespon, F., Ducic, V., Jeansou, E., Occhipinti, G., Helbert, J., Moreaux, G., Godet, P.E.: Ground-based GPS imaging of ionospheric postseismic signal. Planetary and Space Science 54, 528-540 (2006)

2. Błeçki, J., Parrot, M., Wronowski, R.: Studies of the electromagnetic field variations in ELF frequency range registered by DEMETER over the Sichuan region prior to the earthquake. Intern. J. of Remote Sensing 31(13), 3615-3629 (2010)

3. Malmstrom, J.: Robust Navigation with GPS/INS and Adaptive Beamforming, Swedish Defence Research Agency (2003)

4. Fenn, A.J.: Adaptive Antennas and Phased Arrays for Radar and Communications. Artech House, Boston (2008)

5. Borre, K., Akos, D.M., Bertelsen, N., Rinder, P., Jensen, S.H.: A software-defined GPS and GALILEO receiver: A single frequency approach. Birkhauser, Boston (2007)

6. Sainati, R.A.: CAD of micro strip antenna for wireless applications. Artech House, Inc. (1996)

7. Gardiol, F.: Design and layout of micro strip structures. Proceedings IEEE 35(3), 145-157 (1988)

8. Visser, H.: Array and phased array antenna. Wiley, UK (2005)

9. Ghosh, C.K., Parui, S.K.: Design, Analysis and Optimization of A Slotted Microstrip Patch Antenna Array at Frequency 5.25 GHz for WLAN-SDMA System. International Journal on Electrical Engineering and Informatics 2(2), 106-110 (2010)

10. Rogstad, D.H., Mileant, A., Pham, T.T.: Antenna arraying techniques in the deep space network.JPL deep space communications and navigation series. Wiley (2003) 